\title{
WHY MONITOR ACOUSTIC EMISSIONS DURING NANOMECHANICAL TESTS?
}

\author{
RADim ČTVRTLíKK ${ }^{a, *}$, LuKÁŠ VÁCLAVEK ${ }^{b}, J_{A N}$ TOMÁŠTíK ${ }^{c}$ \\ ${ }^{a}$ Institute of Physics of the Czech Academy of Sciences, Joint Laboratory of Optics of Palacky University and \\ Institute of Physics AS CR, Olomouc, Czech Republic, 17. listopadu 50a, 77207 Olomouc, Czech Republic \\ ${ }^{b}$ Joint Laboratory of Optics of Palacky University and Institute of Physics of the Czech Academy of Sciences, \\ Faculty of Science, Palacky University, 17. listopadu 12, 77146 Olomouc, Czech Republic \\ ${ }^{c}$ Regional Centre of Advanced Technologies and Materials, Joint Laboratory of Optics of Palacky University and \\ Institute of Physics of the Czech Academy of Sciences, Faculty of Science, Palacky University, 17. listopadu 12, \\ 77146 Olomouc, Czech Republic \\ * corresponding author: ctvrtlik@fzu.cz
}

\begin{abstract}
Acoustic Emissions (AE) monitoring has been proved as an effective non-destructive technique at the macro scale. Nevertheless, it may also be employed at nano/micro scale during nanomechanical and nanotribological testing. Local mechanical properties of surfaces or micro object are routinely explored using nanoindentation, scratch test or dynamic impact tests that are evaluated based on analysis of depth-load-time records or microscopic observation of residual indents, scratch grooves or impact craters, respectively. Although these approaches have been proven to be sufficient in most cases for a variety of materials, there are many situations where they do not provide sufficient information for a complex understanding of the deformation response. On the other hand, analysis of AE signals generated during these tests may provide valuable complementary information and provide some insight into the dynamics of phenomena like cracking, phase transitions, plastic instabilities, etc. That means that the analysis of acoustic emissions provides a better understanding and more complex interpretation of the results obtained by the above-mentioned tests. The strength of the AE based method is demonstrated for optical thin films deposited on glass tested via scratch test. Combination of $\mathrm{AE}$ and nanoindentation will be presented for various types of glasses. The complementary analysis of the time and frequency domain will draw the potential of the AE extended approach. Especially dynamics of the failure and fracture processes will be distinguished and elucidated. In both cases the analysis of $\mathrm{AE}$ events will be correlated with standard approaches.
\end{abstract}

KEYWORDS: Acoustic emission, indentation, scratch test, thin films.

\section{INTRODUCTION}

Surfaces play an essential part of our everyday lives as they are involved in most of the interactions. There have been developed a wide variety of methods for surface modification based on either their compositional/structural modification or deposition of a surface layer. A tremendous effort has been devoted especially to thin films and coatings technology that allows tailoring of surface properties via numerous PVD (Physical Vapor Deposition), CVD (Chemical Vapor Deposition) and PACVD (Plasma Assisted Chemical Vapor Deposition) methods. In fact, the thin film technology has become an inherent part of almost any manufacturing process. Since the fabrication processes often operate under the non-thermodynamic conditions the physical properties of the films may substantially differ from bulk analogs with the same chemical composition, if even exist. Therefore, the exploration of their physical or chemical properties is of great importance in order to understand their nature and practical limits as well as ways of their modifications.
Regardless of the primary role of the surface property (optical, electrical, protective, lubricating, wetting, catalytic, etc.) predetermined by their application, the surface mechanical durability is vital for a long lifetime of the component/device. Besides, surfaces are often exposed to external stresses during their fabrication. Therefore, reliable testing of mechanical properties is essential for their research and development as well as industrial production. This is very true especially in optics and electronics where most of the surfaces are coated with thin films or multi-layered structures [1, 2,

Probably the most important tests are nanoindentation and scratch test based on quasistatic mechanical contact between the diamond indenter and the tested surface [3]. Dynamic tests are also available at nano/micro scale. Although these tests have been improved and optimized for years for testing of most classes of materials (metals, ceramics, polymers, biomaterials) in various forms (thin films, coatings, composites, etc.), there has been always a pursuit of increasing the information content obtained during these tests. Since the standardly used nano/micro- 
a)

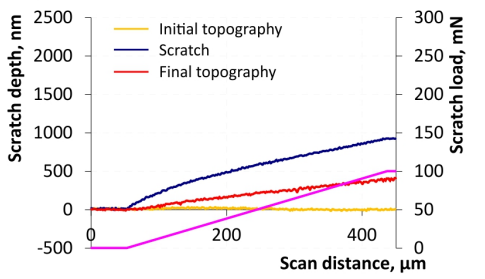

$100 \mathrm{mN}$

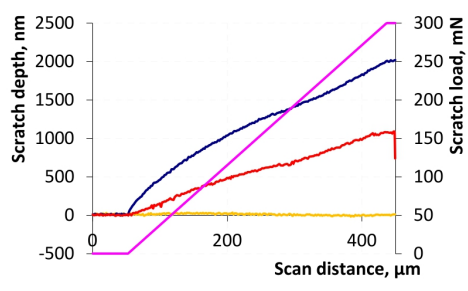

$300 \mathrm{mN}$ b)

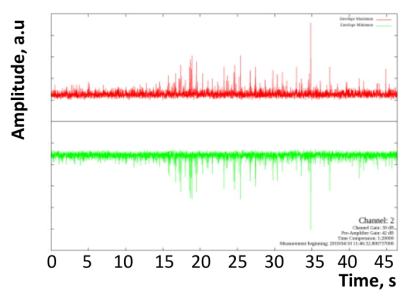

discrete AE

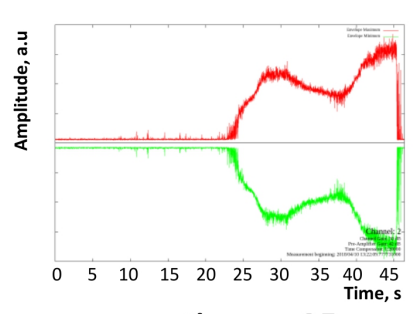

continuous $\mathrm{AE}$ c)

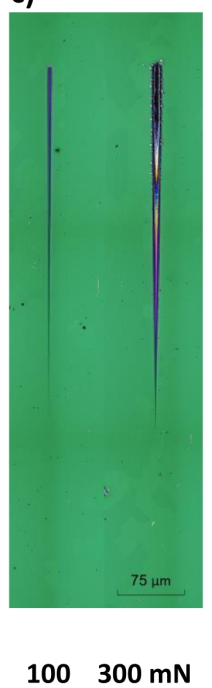

d)

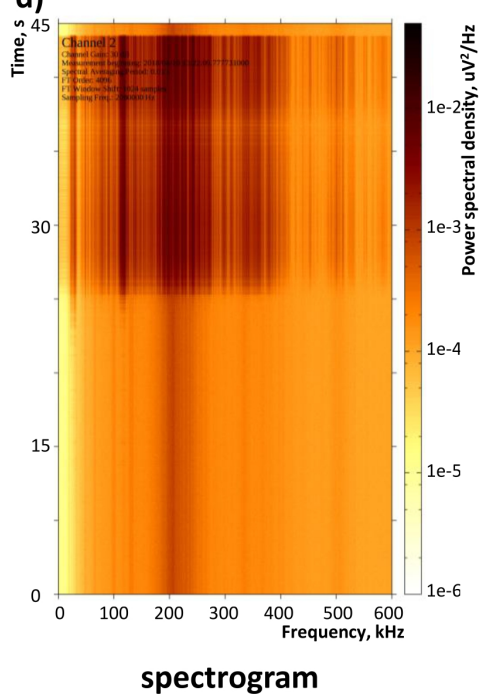

Figure 1. Scratch testing of $\mathrm{TiO}_{2}$ film on glass substrate performed up to 100 and $300 \mathrm{mN}$ a) depth-load-distance records b) AE envelopes c) residual scratch grooves and d) spectrogram for the $300 \mathrm{mN}$ test.

mechanical/tribological tests are based on the analysis of depth-load-time record, which potential is inevitably limited and probably has approached its limit, new techniques are sought. In this perspective, proper application of the acoustic emission method to the nano/micro scale testing seems to be very promising as it proved to be irreplaceable at macro scale due to its ability to reveal the deformation dynamic, subsurface damage, defective area localization, real time monitoring etc.

Acoustic emission might be defined as the phenomenon of transient elastic waves radiation in solids that occurs during a dynamic reconstruction of its internal structure. This may be represented by crack formation, plastic deformation, phase transition, delamination or debonding as typical examples of material acoustic emission. Besides, the mechanical AE is also distinguished and is connected with the mechanical origin (leakage, friction, impact etc.).

The mechanical loading of a material may lead to its structural changes and in turn to generation of elastic stress waves. Consequently, small surface displacements of a material can be detected. This effect is especially strong when the accumulated elastic energy is released rapidly. In practice, the AE method is based on the mechanism described above and is used for example for structural health monitoring, quality control, system feedback, process monitoring, etc.

\section{INSTRUMENTATION}

Nano/micro-mechanical tests were performed using a fully calibrated NanoTest instrument (MicroMaterials, UK) at room temperature. Berkovich indenter was used for indentation, while Rockwell indenter with nominal radius of $10 \mu \mathrm{m}$ was used for scratch testing. The samples were loaded on the custom built AE holder based on dedicated piezo-element and inbuilt pre-amplifier. The glass samples were fixed using the special low melting point wax; though for $\mathrm{AE}$ experiment also super glue can be used.

AE signals were monitored and recorded during the whole test using the ZEDO system (Dakel, Czech Republic) featuring maximum sampling frequency of $10 \mathrm{MHz}$. The high dynamic range and the broad frequency range of $2 \mathrm{MHz}$ allow exploring strong as well as weak signals with sub-microsecond resolution.

\section{Results And Discussion}

\subsection{Optical THIN Films}

Scratch test is considered as a standard method used for exploration of adhesion and cohesion of thin films and coating. During the test, the diamond tip is pulled over the surface while the normal force applied on the indenter is increasing. The goal of the test is to find the critical loads representing the onset of specific failures. There are various ways how to find these loads based on either i) visual observation of the residual groove or ii) analysis of depth-loaddistance record or evaluation of the iii) friction probe and iv) AE signal. It should be noted that especially microscopic analysis is mostly used in practice.

It has been shown that for reliable nano/micro scratch test evaluation the combination of all the above mentioned techniques should be used [4]. As a benefit it is also possible to get insight into the dynamics of the failure process [5]. In practice, the AE signal envelope, that is in fact a compressed signal, has proved to be reliable source of information. Nevertheless, the thorough evaluation of the sampled AE signal considerably enhances the AE analysis and allows distinguishing between different failure types (cracking, chipping or delamination etc.) and/or the initial and catastrophic film/substrate failures. In general the AE signal may be analysed in both the time and frequency domain. The visual representation 
a)

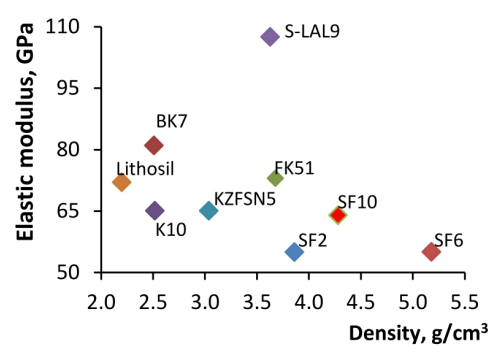

b)

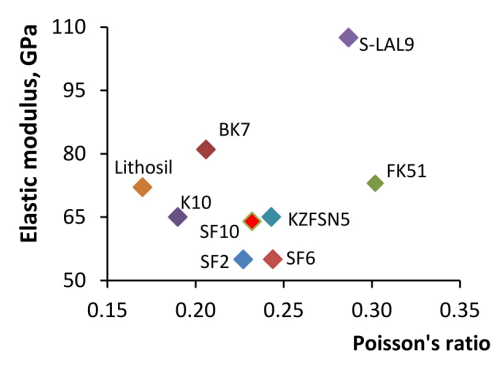

c)

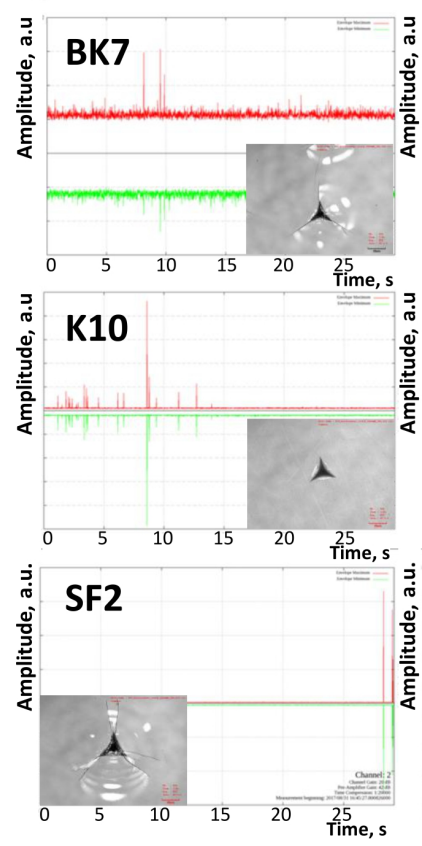

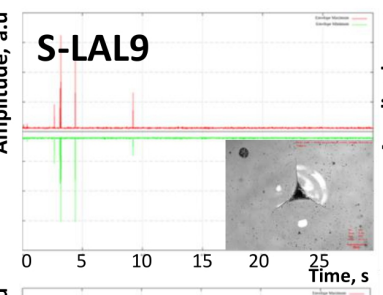
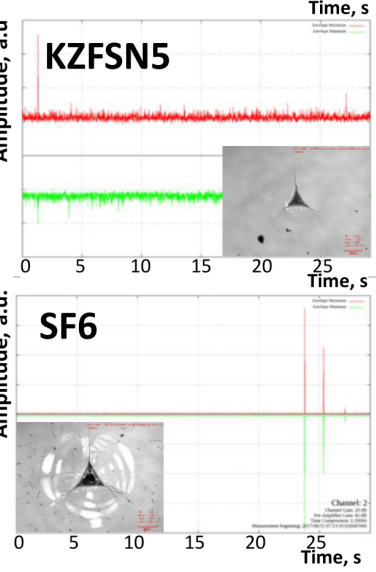
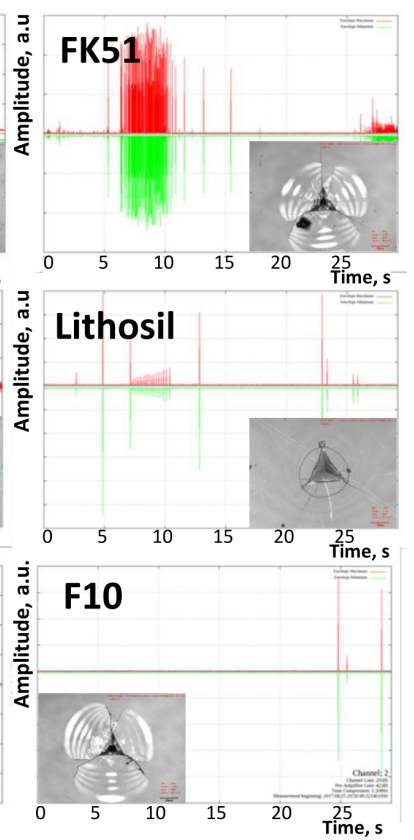

Figure 2. Overview of $\mathrm{a}, \mathrm{b}$ ) elastic properties and density, and c) residual crack patterns together with AE records for various glasses tested with pyramidal Berkovich indenter.

of the spectrum of frequencies as a function of time for the whole AE signal is called spectrogram.

In some cases, the $\mathrm{AE}$ is the only method able to unambiguously determine the film and substrate damage, like in case of optical $\mathrm{TiO}_{2}$ thin film with thickness of $280 \mathrm{~nm}$ deposited on soda-lime silicate glass substrate, see Fig. 1. The discrete AE envelope reflects the film cracking, while the continuous AE reveals total film wipe off and glass damage. The continuous character of the $\mathrm{AE}$ envelope reflects consecutive damage of the glass substrate in the form of successive $\mathrm{AE}$ events. It should be noted that depth-load-distance as well as optical observation of the residual scratch groove themselves do not provide sufficient information for reliable evaluation of the test. Based on the AE record analysis the critical load of $18 \mathrm{mN}$ was evaluated.

Acoustic emission technique is not only useful addon for the scratch test. It rather represents an important and reliable approach how to detect the very first cracking in the film/substrate system. Furthermore, it offers a real-time detection of the subsurface damage in the very convenient way.

\subsection{OPTICAL GLASS}

Nanoindentation (Depth sensing indentation) is a contact based technique widely used for evaluation of mechanical properties at small scales. During a typical test a diamond tip of known geometry is pressed into the sample surface, while the indentation load and depth are continuously recorded. Hardness and elastic modulus are measured in most cases, but other phenomena like pressure-induced phase transformations [6, 7], incipient plasticity or time dependent effects can be studied. Furthermore, it is possible to perform such test at elevated temperatures and study temperature stability of the material or temperature related phenomena $[8]$.

Nanoindentation is very effective for hardness and modulus evaluation provided several assumptions are fulfilled. For example absence of cracking, fully developed plastic zone, no substrate effect, thermal stability etc. Ignoring these facts might lead to underestimation of hardness and elastic modulus values. Nevertheless, since normal load is applied in a very controlled way during the indenter penetration the indentation test is well suited for studying the glass surface mechanical durability as conditions for stable crack growth are formed. It is well known that sharp point contact is one of the most often failure mode in glass, especially in chemically tempered glass. The glass surface damage induced by a sharp point contact is a serious problem in practice and still not fully understood comprehensive issue encompassing fracture mechanics and glass science.

Therefore nanoindentation using sharp indenter is a very convenient way of testing this phenomenon as diamond tip can be used to mimic the damage from sharp contact. What is more, this approach allows using of different tip geometries (pyramidal and spherical with different contact angles and radii, respectively) to simulate different loading conditions (load, loading rate etc.).

In this study we indented 9 samples of optical glasses with different elastic moduli, Poissons ratios and densities with Berkovich indenter, see Fig. 2 Glasses were selected in order to cover as broad as possible range of elastic modulus (55-108) GPa, Poissons ratio (0.170-0.302) and density $(2.20-5.18) \mathrm{g} / \mathrm{cm}^{3}$. Infor- 
a)
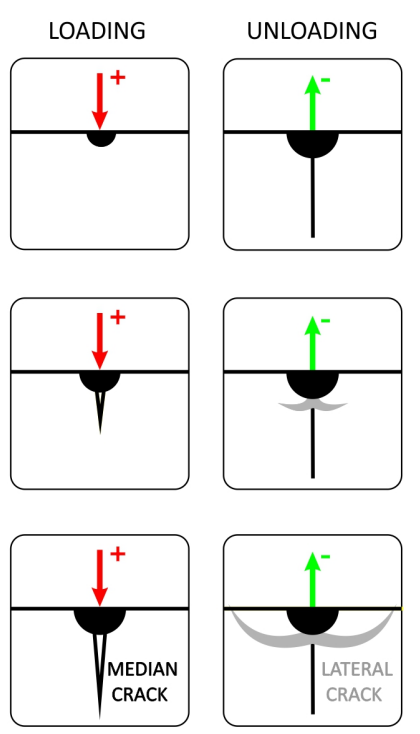

b)

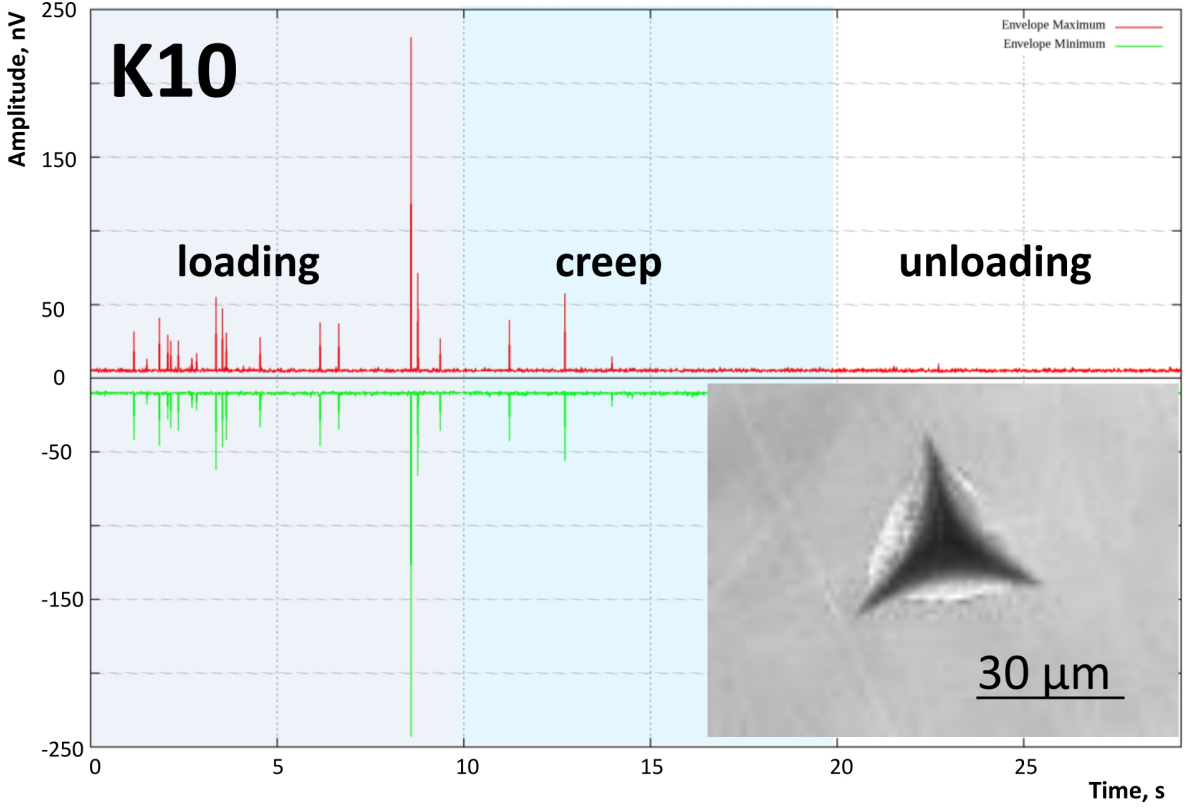

FIgURE 3. Crack evolution during indentation with sharp indenter a) theoretical diagram (based on a drawing by Lawn 9] ) b) Schott optical glass K10 - AE envelope and detail od residual indent made by Berkovich indenter at $20 \mathrm{~N}$.

a)

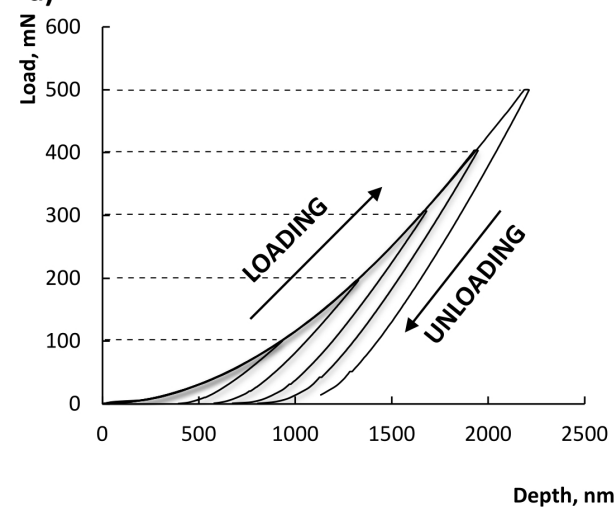

b) $400 \mathrm{mN}$
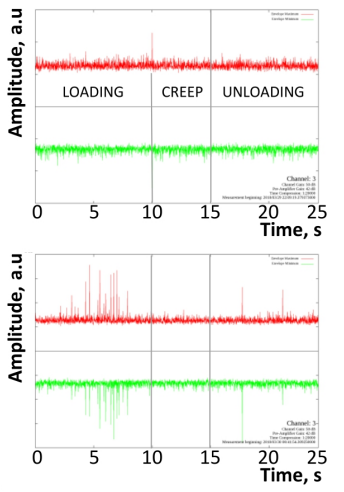

$500 \mathrm{mN}$
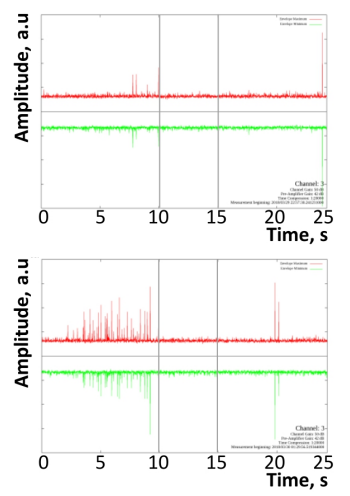

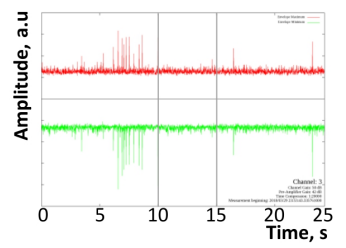

c)

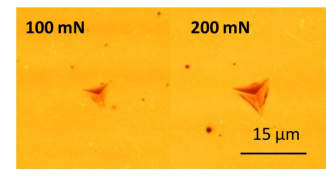

FIgURE 4. Indentation testing of fused silica from 100 to $500 \mathrm{mN}$ a) indentation curves b) AE records and c) residual indents for 100 and $200 \mathrm{mN}$.

mation about the properties of glasses used in this study was obtained from the datasheets provided by the manufacturers (Shott, Ohara, Sumita).

Different types of glasses can crack during both the loading and the unloading sequence with various crack patterns as shown in Fig. 2. It can be clearly seen that the resistivity against crack formation and propagation differs extensively. Different types of cracks and their combinations can be visually distinguished. Analysis of the AE envelopes might then assigns the specific types of cracks to either loading (first 10 seconds) or unloading (last 10 seconds) stage of the indentation process, see Fig. 3 a. For example the lateral cracks are formed during the unloading when the elastically deformed material recovers. The AE peaks during the loading can be ascribed to the formation of the so called median cracks that originate beneath the indenter and are closed during unloading. It should be noted, that especially in glasses the median cracks interfere with radial cracks emanating from the residual imprint corners and form so called median/radial cracks.

Microscopic observation of the residual indents for the optical glass K10, a classic glass type with lead oxide, did not reveal any traces of cracking, instead pile up was observed as can be seen in inset in Fig. 3p. On the other hand, the AE record clearly points out on structural changes beneath the indenter taking place during loading and dwell period (creep). This implies that median cracking is the main cracking mode. It should be noted that median cracks propagate perpendicular to the surface and have stronger effect on glass strength in comparison to lateral cracks spreading more or less parallel to the surface. When 
lateral crack reaches the surface then large fragments might delaminate causing material loss.

\subsection{FUSED SILICA}

Use of AE detection system during indentation of fused silica revealed marked cracking already at $100 \mathrm{mN}$, see Fig. 4 This finding might have serious consequences on reliability of calculated elastic modulus values as fused silica is routinely used as a calibration material for assessment of indenter shape function. Cracking of the material is inconsistent with the assumption of the elastic-plastic loading and purely elastic unloading [10. It should be noted that no traces of cracking are detectable on indentation curves.

\section{Conclusions}

Information provided by the nano/micromechanical/tribological tests can be easily extended by the use of acoustic emission detection system. New and unique information on material deformation behavior can be obtained in a non-destructive way. Combination of depth-load-time records (indentation curve / scratch record / impulse depth) and images of residual indents / scratch grooves / impact craters with AE record provides a better insight in to the deformation dynamics.

Acoustic emission broadens the possibilities in scratch test evaluation as it allows detection of the very first subsurface cohesion and/or adhesion failures otherwise inaccessible. In specific cases it is also possible to separate film and substrate failures.

It was shown that use of $\mathrm{AE}$ and indentation is very effective in exploring mechanical durability and cracking resistivity of optical glass surfaces against sharp point contact. Different cracking patterns together with different AE records were observed. However, no unambiguous relationship between crack type and glass elastic properties and density was observed under used experimental conditions.

\section{ACKNOWLEDGEMENTS}

The authors gratefully acknowledge the support by the Project TH03020245 of the Technology Agency of the Czech Republic and the Operational Programme Research, Development and Education, Projects Nos. CZ.02.1.01/0.0/0.0/17_049/0008422 and CZ.02.1.01/0.0/0.0/16 019/0000754 of the Ministry of Education, Youth and Sports of the Czech Republic. This work was also supported by the project IGA_PrF_2019_008 of Palacky University.

\section{REFERENCES}

[1] L. Martinu, D. Poitras. Plasma deposition of optical films and coatings. Journal of Vacuum Science \&5 Technology A Vacuum Surfaces and Films 18:2619-2645, 2000. DOI:10.1116/1.1314395.

[2] M. Mozetič, A. Vesel, G. Primc, et al. Recent developments in surface science and engineering, thin films, nanoscience, biomaterials, plasma science, and vacuum technology. Thin Solid Films 660:120 - 160, 2018. DOI:10.1016/j.tsf.2018.05.046.

[3] A. Gouldstone, N. Chollacoop, M. Dao, et al. Indentation across size scales and disciplines: Recent developments in experimentation and modeling. Acta Materialia 55(12):4015 - 4039, 2007. DOI:10.1016/j.actamat.2006.08.044

[4] J. Tomastik, R. Ctvrtlik, M. Drab, J. Manak. On the importance of combined scratch/acoustic emission test evaluation: $\mathrm{SiC}$ and $\mathrm{SiCN}$ thin films case study. Coatings 8(5), 2018. DOI:10.3390/coatings8050196

[5] J. Tomastik, R. Ctvrtlik, T. Ingr, et al. Effect of nitrogen doping and temperature on mechanical durability of silicon carbide thin films. Scientific Reports 8, 2018. DOI:10.1038/s41598-018-28704-3

[6] V. Kulikovsky, V. Vorlicek, P. Bohac, et al. Mechanical properties of amorphous and microcrystalline silicon films. Thin Solid Films 516(16):5368-5375, 2008. DOI:10.1016/j.tsf.2007.07.047.

[7] V. Kulikovsky, R. Ctvrtlik, V. Vorlicek, et al. Mechanical properties and structure of $\mathrm{TiO}_{2}$ films deposited on quartz and silicon substrates. Thin Solid Films 542:91 - 99, 2013. DOI:10.1016/j.tsf.2013.06.070

[8] R. Ctvrtlik, M. Al-Haik, V. Kulikovsky. Mechanical properties of amorphous silicon carbonitride thin films at elevated temperatures. Journal of Materials Science 50, 2015. DOI:10.1007/s10853-014-8715-0

[9] B. Lawn, M. Swain. Microfracture beneath point indenters in brittle solids. Journal of Materials Science 10:113-122, 1975. DOI:10.1007/BF00541038

[10] A. C. Fischer-Cripps. Nanoindentation. Springer, New York, NY, 3rd edn., 2011. DOI:10.1007/978-1-4419-9872-9 